\title{
Color glass condensate formalism, Balitsky-JIMWLK evolution, and Lipatov's high energy effective action
}

\author{
Martin Hentschinski \\ Departamento de Actuaria, Física y Matemáticas, Universidad de las Américas Puebla, \\ Santa Catarina Martir, 72820 Puebla, Mexico
}

(Received 21 February 2018; published 26 June 2018)

\begin{abstract}
We investigate the question whether Lipatov's high energy effective action is capable of reproducing quark and gluon propagators that resum the interaction with a strong background field within high energy factorization. Such propagators are frequently employed in calculations within the color glass condensate formalism, in particular when considering scattering of a dilute projectile on a dense target nucleus or nucleon. We find that such propagators can be obtained from the high energy effective action, if a special parametrization of the gluonic field is used, first proposed by Lipatov in the original publication on the high energy effective action. The obtained propagators are used to rederive from the high energy effective action the leading order Balitsky, Jalilian-Marian, Iancu, McLerran, Weigert, Leonidov, Kovner evolution equation in covariant gauge. As an aside, our result confirms the definition of the Reggeized gluon as the logarithm of an adjoint Wilson line, proposed in the literature.
\end{abstract}

DOI: 10.1103/PhysRevD.97.114027

\section{INTRODUCTION}

The color glass condensate (CGC) formalism is an effective field theory approach to quantum chromodynamics (QCD) at small $x$ where gluon densities in the nucleus or proton are large. With $x$ the ratio of the hard scale $M^{2}$ of a certain hard process and $s$ the center-of-mass energy squared, the limit $x \rightarrow 0$ at fixed $M^{2}$ corresponds to the perturbative Regge limit of QCD. In such a scenario, the smallness of the strong coupling $\alpha_{s}\left(M^{2}\right) \ll 1$ can be compensated by logarithms in $x, \alpha_{s}\left(M^{2}\right) \ln 1 / x \sim 1$, which requires the resummation of terms $\left(\alpha_{s}\left(M^{2}\right) \ln 1 / x\right)^{n}$ to all orders. For perturbative scattering amplitudes, such a resummation is achieved at leading [1,2] and next-toleading order [3] by the Balitsky-Fadin-Kuraev-Lipatov (BFKL) evolution equation. Even though BFKL evolution is successfully applied to the description of collider data at currently accessible center-of-mass energies (see, e.g., [4]), the powerlike rise of the gluon distribution predicted by BFKL evolution will eventually drive cross sections to a region of phase space, where parton densities are no longer perturbative; BFKL evolution will therefore break down in such a regime. Instead, it is more appropriate to treat the

\footnotetext{
* Corresponding author. martin.hentschinski@udlap.mx

Published by the American Physical Society under the terms of the Creative Commons Attribution 4.0 International license. Further distribution of this work must maintain attribution to the author(s) and the published article's title, journal citation, and DOI. Funded by SCOAP .
}

hadron or nucleus as a coherent color field rather than a collection of incoherent and individual partons. This is the region of phase space that is addressed by the initially mentioned CGC; see [5] for a review. At the classical level, the CGC generalizes scattering via exchange of a single gluon to multiple gluon exchanges within high energy factorization. Including, furthermore, quantum effects, one arrives at a resummation of logarithms in $1 / x$, generalizing BFKL evolution to the case of large gluon densities. The resulting Balitsky, Jalilian-Marian, Iancu, McLerran, Weigert, Leonidov, Kovner (Balitsky-JIMWLK) evolution [6-10] provides finally an evolution equation for Wilson lines that sum up the strong gluonic field in the target.

In the present article we discuss Lipatov's high energy effective action [11,12] and its relation to the above mentioned formulation of a CGC effective theory. One of the main advantages of Lipatov's high energy effective action is that it provides a gauge invariant factorization of QCD amplitudes in the high energy limit through introducing a new type of field, i.e., the Reggeized gluon. Using this effective action it has been possible to both reproduce and derive a number of next-to-leading order (NLO) results, most notable the calculation of NLO correction to forward jet production without $[13,14]$ and with rapidity gap [15], the gluon Regge trajectory up to two loops [16], and the NLO kernel of the Bartels-KwiecinskiPraszalowicz evolution equation [17] (see also the review [18]); for the determination of NLO corrections for Reggeized quarks, see [19]. The description of scattering amplitudes for multiple Reggeized gluon exchange has also been studied by a number of authors; see, e.g., [20-22]. At 
the same time the ability of the Balitsky-JIMWLK evolution to reproduce scattering amplitudes with multiple Reggeized gluon states has been demonstrated for various cases (see, e.g., [23,24]), hinting at a possible equivalence of both formalisms. Furthermore the color glass condensate formalism and the high energy effective action have been compared directly at the level of the Lagrangian; see, e.g., $[8,25,26]$. In particular, the authors of Ref. [26] demonstrate that it is possible to reproduce the classical gluon fields of the CGC approach from the Lipatov's high energy effective action.

Instead of comparing the two approaches on the level of the resulting effective Lagrangians, we take here a pragmatic approach and attempt to answer the question whether Lipatov's high energy effective action can be used to reproduce the quark and gluon propagators in the presence of a strong gluonic field. Such propagators are one of the core elements in calculations of the scattering of dilute projectiles on dense targets within the color-glass-condensate formalism. We find that this can be indeed achieved by choosing a special parametrization of the gluonic field already proposed in [11]. Moreover, since Lipatov's high energy effective action provides a gauge invariant factorization of QCD amplitudes in the high energy limit, the resulting propagators are not restricted to a certain gauge, such as the light-cone gauge. The obtained propagators furthermore allow one to rederive leading order BalitskyJIMWLK evolution directly from Lipatov's high energy effective action. As an aside, our result confirms that the definition of the Reggeized gluon as the logarithm of adjoint Wilson lines, proposed in [27], is consistent with Lipatov's high energy effective action.

The outline of this paper is as follows. Section II provides a short summary of Lipatov's high energy effective action. Section III introduces the special parametrization of the gluonic field proposed in [11] and demonstrates how it can be used to derive resummed partonic propagators in the presence of a strong Reggeized gluon field. Section IV contains a comparison of our result with the literature. Section V presents a derivation of Balitsky-JIMWLK evolution from Lipatov's high energy effective action. In Section VI we summarize our results and draw our conclusions. Some details of the calculations are summarized in two appendixes.

\section{THE HIGH-ENERGY EFFECTIVE ACTION}

Within the framework provided by Lipatov's effective action [11,12], QCD amplitudes are in the high energy limit decomposed into gauge invariant subamplitudes, which are localized in rapidity space. The effective Lagrangian then describes the coupling of quarks $(\psi)$ and gluon $\left(v_{\mu}\right)$ fields to a new degree of freedom, the Reggeized gluon field $A_{ \pm}(x)$. The latter is introduced as a convenient tool to reconstruct the complete QCD amplitudes in the high energy limit out of the subamplitudes restricted to small rapidity intervals. Lipatov's effective action is obtained by adding an induced term $S_{\text {ind }}$. to the QCD action $S_{\mathrm{QCD}}$,

$$
S_{\mathrm{eff}}=S_{\mathrm{QCD}}+S_{\mathrm{ind}}
$$

where the induced term $S_{\text {ind }}$. describes the coupling of the gluonic field $v_{\mu}=-i t^{a} v_{\mu}^{a}(x)$ to the Reggeized gluon field $A_{ \pm}(x)=-i t^{a} A_{ \pm}^{a}(x)$, with $t^{a}$ a $\mathrm{SU}\left(N_{c}\right)$ generator in the fundamental representation, $\operatorname{tr}\left(t^{a} t^{b}\right)=\delta^{a b} / 2$. For the definition of light-cone directions we follow the conventions established in the original publication [11],

$$
k^{ \pm}=n^{ \pm} \cdot k=n_{\mp} \cdot k=k_{\mp},
$$

with $n^{ \pm} \cdot n^{\mp}=2$ and $\left(n^{ \pm}\right)^{2}=0$. This implies the following Sudakov decomposition of a four momentum

$$
k=\frac{k^{+}}{2} n^{-}+\frac{k^{-}}{2} n^{+}+\boldsymbol{k}=\frac{k_{-}}{2} n_{+}+\frac{k_{+}}{2} n_{-}+\boldsymbol{k} .
$$

Note that transverse momenta and coordinates will be denoted by bold letters. Furthermore

$$
\partial_{ \pm} x^{ \pm}=2, \quad \partial_{\mp} x^{ \pm}=0 .
$$

High energy factorized amplitudes reveal strong ordering in plus and minus components of momenta, which leads to the following kinematic constraint obeyed by the Reggeized gluon field:

$$
\partial_{+} A_{-}(x)=0=\partial_{-} A_{+}(x) .
$$

Even though the Reggeized gluon field is charged under the QCD gauge group $\mathrm{SU}\left(N_{c}\right)$, it is defined to be invariant under local gauge transformation $\delta_{L} A_{ \pm}=0$. With the local gauge transformations of gluon and quark fields given by

$\delta_{\mathrm{L}} v \mu=\frac{1}{g}\left[D_{\mu}, \chi_{L}\right], \quad \delta_{\mathrm{L}} \psi=-\chi_{L} \psi, \quad D_{\mu}=\partial_{\mu}+g v_{\mu}$,

where $D_{\mu}$ denotes the covariant derivative and $\chi_{L}$ the parameter of the local gauge transformations that decreases for $x \rightarrow \infty$, the Reggeized gluon fields are invariant under local gauge transformations,

$$
\delta_{\mathrm{L}} A_{ \pm}=\frac{1}{g}\left[A_{ \pm}, \chi_{L}\right]=0 .
$$

The kinetic term and the gauge invariant coupling of the Reggeized gluon field to the QCD gluon field are provided by the induced term

$$
\begin{aligned}
S_{\text {ind }}= & \int \mathrm{d}^{4} x\left\{\operatorname{tr}\left[\left(T_{-}[v(x)]-A_{-}(x)\right) \partial_{\perp}^{2} A_{+}(x)\right]\right. \\
& \left.+\operatorname{tr}\left[\left(T_{+}[v(x)]-A_{+}(x)\right) \partial_{\perp}^{2} A_{-}(x)\right]\right\} .
\end{aligned}
$$


The functionals $T_{ \pm}[v]$ can be obtained from the following operator definition:

$$
\begin{aligned}
T_{ \pm}[v] & =-\frac{1}{g} \partial_{ \pm} \frac{1}{1+\frac{g}{\partial_{ \pm}} v_{ \pm}} \\
& =v_{ \pm}-g v_{ \pm} \frac{1}{\partial_{ \pm}} v_{ \pm}+g^{2} v_{ \pm} \frac{1}{\partial_{ \pm}} v_{ \pm} \frac{1}{\partial_{ \pm}} v_{ \pm}-\cdots
\end{aligned}
$$

where the integral operator is implied to act on a unit constant matrix from the left. Boundary conditions of the $1 / \partial_{ \pm}$are fixed through

$$
\begin{aligned}
\frac{1}{1+\frac{g}{\partial_{ \pm}} v_{ \pm}}= & \mathcal{P} \exp \left(-\frac{g}{2} \int_{-\infty}^{x^{ \pm}} d x^{\prime \pm} v_{ \pm}\left(x^{\prime}\right)\right) \\
= & 1-\frac{g}{2} \int_{-\infty}^{x^{ \pm}} d x^{\prime \pm} v_{ \pm}\left(x^{\prime}\right) \\
& +\frac{g^{2}}{4} \int_{-\infty}^{x^{ \pm}} d x^{\prime \pm} \int_{-\infty}^{x^{\prime \pm}} d x^{\prime \prime \pm} v_{ \pm}\left(x^{\prime}\right) v_{ \pm}\left(x^{\prime \prime}\right)+\cdots
\end{aligned}
$$

Because of the induced term in Eq. (1), the Feynman rules of the effective action comprise, apart from the usual QCD Feynman rules, the propagator of the Reggeized gluon and an infinite number of so-called induced vertices. The leading order vertices and propagators are summarized in Fig. 1. These induced vertices are special in the sense that they contain only the antisymmetric color-octet sector of the eikonal operator Eq. (9).

While the projection on the color octet sector arises automatically from the induced term due to the combination with the Reggeized gluon field, the antisymmetric color structure [written in terms of $\mathrm{SU}\left(N_{c}\right)$ structure constants only] requires in general the use of a corresponding projector; for an explicit construction see [28]. The original argument given by Lipatov for this projection is based on the observation that in generalized multi-Regge kinematics the values of the operator $\partial_{ \pm}$ acting on a gluonic field is never zero for the vertices arising from Eq. (8), since the resulting light-cone momenta are proportional to large center-of-mass energies of clusters of particles significantly separated in rapidity. In particular,

$$
\frac{1}{\partial_{ \pm}} \tilde{v}_{ \pm}(p)=\frac{i}{p_{ \pm}} \tilde{v}_{ \pm}(p)
$$

with $p_{ \pm} \neq 0$ where $\tilde{v}_{ \pm}(p)$ denotes the Fourier transform of the gluonic field $v(x)$; this is especially true for the case of real particle production within the generalized multiRegge kinematics, which initiated the discussion of the formulation of the high energy effective action in [11]. For a more detailed discussion we refer to [29]. With $p_{ \pm} \neq 0$, antisymmetric color structure as given in Fig. 1 arises automatically from the high energy effective action; see also the discussion in [29]. The condition $p_{ \pm} \neq 0$ is, however, at least at first violated in the evaluation of loop integrals, where the $p_{ \pm}$are integrated over all possible values. The projection of [28] implies then the use of the boundary conditions of Eq. (10), with an additional projection for the color structure of the vertices in Fig. 1 on the desired anti-symmetric color octet sector. Corresponding symmetric counterparts are then taken into account by an exchange of multiple Reggeized gluons and the combination of multiple Reggeized gluons and induced vertices; see also the discussion in Appendix A. In the following we use always the pole prescription for induced vertices proposed in [28].

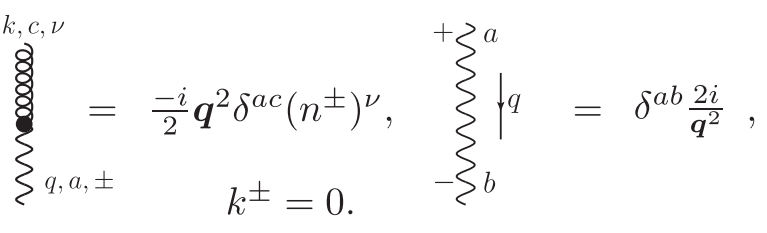

(a)

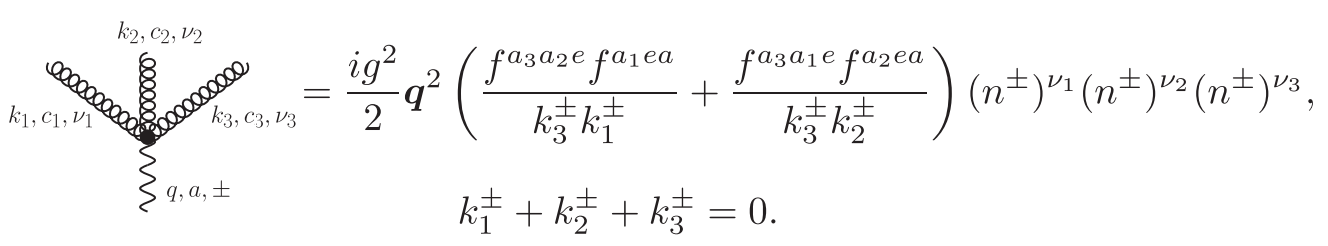

(d)

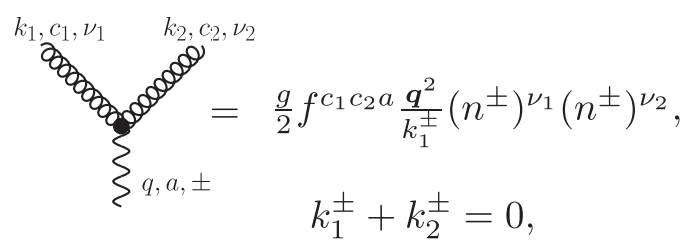

(c)

FIG. 1. Feynman rules for the lowest-order effective vertices of the effective action. Wavy lines denote Reggeized fields and curly lines denote gluons. Note that in comparison with the Feynman rules used in [13-16] we absorb a factor of $1 / 2$ into the vertices, which is compensated by changing the residue of the Reggeized gluon propagator from $1 / 2$ to 2 . 


\section{RESUMMATION OF A STRONG REGGEIZED GLUON FIELD}

In the following we provide a formulation of the high energy effective action that allows for a straightforward resummation of multiple Reggeized gluon exchange in the chase of quasielastic scattering, which is the relevant case for describing scattering of a dilute partonic projectile on a dense target nucleus or proton.

\section{A. A special parametrization of the gluonic field}

The bulk of calculations performed within the framework set by the high energy effective action employs the vertex of Fig. 1(a) that provides a direct transition between a Reggeized gluon field and a conventional QCD gluon. As noted in [11,12], it is possible to avoid the use of such a direct transition vertex, if one performs a shift $v_{ \pm} \rightarrow V_{ \pm}=$ $v_{ \pm}+A_{ \pm}$of the gluonic field in the effective action. ${ }^{1}$ Such a shift has, however, the disadvantage that the gluonic field $v_{ \pm}$transforms like a gauge field under local gauge transformations while the Reggeized gluon field is invariant under such transformations. To avoid such differing transformation properties, the following parametrization of the gluonic field has been proposed in [11]:

$$
\begin{aligned}
V^{\mu}(x)= & v^{\mu}(x)+\frac{n_{+}^{\mu}}{2} U\left[v_{+}(x)\right] A_{-}(x) U^{-1}\left[v_{+}(x)\right] \\
& +\frac{n_{-}^{\mu}}{2} U\left[v_{-}(x)\right] A_{+}(x) U^{-1}\left[v_{-}(x)\right] \\
= & v^{\mu}(x)+\frac{n_{+}^{\mu}}{2} B_{-}(x)+\frac{n_{-}^{\mu}}{2} B_{+}(x),
\end{aligned}
$$

where

$$
B_{ \pm}\left[v_{\mp}\right]=U\left[v_{\mp}\right] A_{ \pm} U^{-1}\left[v_{\mp}\right],
$$

and (inverse) Wilson line operators are defined as

$$
U\left[v_{ \pm}\right]=\frac{1}{1+\frac{g}{\partial_{ \pm}} v_{ \pm}}, \quad U^{-1}\left[v_{ \pm}\right]=1+\frac{g}{\partial_{ \pm}} v_{ \pm} .
$$

Here the integral operators $U$ and $U^{-1}$ act on a unit constant matrix from the left- and right-hand sides, respectively. For the above composite field $B_{ \pm}\left[v_{\mp}\right]$, one finds the following gauge transformation properties:

$$
\begin{aligned}
\delta_{L} B_{ \pm} & =\delta_{L} U\left[v_{\mp}\right] A_{ \pm} U^{-1}\left[v_{\mp}\right]+U\left[v_{\mp}\right] A_{ \pm} \delta_{L} U^{-1}\left[v_{\mp}\right] \\
& =\left[g B_{ \pm}, \chi_{L}\right] .
\end{aligned}
$$

As a consequence the shifted gluonic field Eq. (12) transforms as

\footnotetext{
${ }^{1}$ Such a shift has been used, for instance, in [20,21].
}

$$
\delta V_{ \pm}=\left[D_{ \pm}, \chi\right]+\left[g B_{ \pm}, \chi\right]=\left[D_{ \pm}+g B_{ \pm}, \chi\right] ;
$$

i.e., the field $V_{\mu}$ has consistent gauge transformation properties corresponding to a gauge field. In the following we will use the above parametrization of the gluonic field to expand the high energy effective action for the quasielastic case around the Reggeized gluon field $A_{+}$, which we treat as a strong classical background field $g A_{+} \sim 1$.

\section{B. The effective Lagrangian quadratic in $\boldsymbol{v}_{\boldsymbol{\mu}}$}

In the following we limit ourselves to the quasielastic case where the Lagrangian contains only the induced terms corresponding to the functional $W_{-}[v]$. The second set of induced terms is set aside for the moment. This is sufficient to describe the interaction of a dilute projectile with a target characterized by high parton densities in the high energy limit, where the $A_{+}$will couple through the Reggeized gluon propagator to color charges in the target. To construct the effective action for quasielastic processes, we use the following parametrization of the gluonic field:

$$
V^{\mu}(x)=v^{\mu}(x)+\frac{1}{2}\left(n_{-}\right)^{\mu} B_{+}\left[v_{-}\right]
$$

and consider the following effective action for the quasielastic case:

$$
S_{\text {eff }}^{\text {q.e. }}=S_{\mathrm{QCD}}+S_{\text {ind }}^{\text {q.e. }}
$$

with

$$
S_{\mathrm{QCD}}=\int d^{4} x\left[\operatorname{tr}\left(\frac{1}{2} G_{\mu \nu} G^{\mu \nu}\right)+\bar{\psi}(x)(i \not D) \psi(x)\right],
$$

where $G_{\mu \nu}=\frac{1}{g}\left[D_{\mu}, D_{\nu}\right]$ and

$$
S_{\text {ind }}^{\text {q.e. }}=\int d^{4} x \operatorname{tr}\left(\left\{W_{-}[v]-A_{-}(x)\right\} \partial^{2} A_{+}(x)\right] \text {. }
$$

Keeping fields $A_{+}$to all orders and expanding in quantum fluctuations $v_{\mu}$ and $\psi, \bar{\psi}$ to quadratic order we obtain

$$
S_{\text {eff }}^{\text {q.e. }}=\int d^{4} x\left[\mathcal{L}_{0}+\mathcal{L}_{1}-\operatorname{tr}\left(A_{-} \partial^{2} A_{+}\right)\right]+\mathcal{O}\left(v_{\mu}^{3}\right),
$$

with the kinetic term of the gluonic and quark field

$$
\mathcal{L}_{0}=\operatorname{tr}\left(-v^{\mu}\left[g_{\mu \nu} \partial^{2}-\partial_{\mu} \partial_{\nu}\right] v^{\nu}\right)+\bar{\psi} i \not \psi \psi
$$

and the quadratic terms that describe the interaction with the Reggeized gluon field, 


$$
\begin{aligned}
\mathcal{L}_{1}= & g \cdot\left\{\frac{i}{2} \bar{\psi} h_{-} A_{+} \psi+\operatorname{tr}\left[\partial_{-} v_{\mu}\left[A_{+}, v^{\mu}\right]+2 \partial_{\mu} v_{-}\left[v^{\mu}, A_{+}\right]\right.\right. \\
& \left.\left.+\partial^{2} v_{-}\left[\left(\frac{1}{\partial_{-}} v_{-}\right), A_{+}\right]-v_{-}\left(\frac{1}{\partial_{-}} v_{-}\right) \partial^{2} A_{+}\right]\right\} .
\end{aligned}
$$

Since we assume that the Reggeized gluon field couples to high partonic densities in the target, we have $g A_{+} \sim 1$; the term $\mathcal{L}_{1}$ is therefore of the same order as $\mathcal{L}_{0}$. The term $\operatorname{tr}\left(A_{-} \partial^{2} A_{+}\right)$provides the kinetic term of the Reggeized gluon field, which is only needed to connect the $A_{+}$field to, e.g., the target.

\section{Parton-parton-Reggeized gluon vertices}

The above Lagrangian $\mathcal{L}_{1}$ allows now for the straightforward determination of the quark-quark-Reggeized gluon (QQR) and gluon-gluon-Reggeized gluon (GGR) vertex. Keeping an explicit dependence on the Reggeized gluon field, we find for quarks,

$$
\begin{aligned}
\sum_{i, \alpha}^{p} \underbrace{r}_{j, \beta} & =-i g t_{j i}^{c} \Gamma_{\beta \alpha}(r, p) \int d^{4} z e^{-i z \cdot(p-r)} A_{+}^{c}(z), \\
\Gamma_{\beta \alpha}(r, p) & =-\frac{1}{2} \hbar_{\alpha \beta}^{+},
\end{aligned}
$$

which coincides with the expression used, e.g., in [13]. For gluons one obtains instead

$$
\begin{aligned}
\underset{a, \mu}{p} \underset{\sum_{0,+}}{\stackrel{r}{r}} & =-i g T_{b a}^{c} \Gamma^{\nu \mu}(r, p) \int d^{4} z e^{-i z \cdot(p-r)} A_{+}^{c}(z), \\
\Gamma_{+}^{\nu \mu}(r, p) & =p^{+} g^{\mu \nu}-\left(n^{+}\right)^{\mu} p^{\nu}-\left(n^{+}\right)^{\nu} r^{\mu}+\frac{r \cdot p}{p^{+}}\left(n^{+}\right)^{\mu}\left(n^{+}\right)^{\nu} \\
& =p^{+} g_{\perp}^{\mu \nu}-\left(n^{+}\right)^{\mu} \boldsymbol{p}^{\nu}-\left(n^{+}\right)^{\nu} \boldsymbol{r}^{\mu}-\frac{\boldsymbol{r} \cdot \boldsymbol{p}}{p^{+}}\left(n^{+}\right)^{\mu}\left(n^{+}\right)^{\nu},
\end{aligned}
$$

with $T_{a b}^{c}=-i f^{a b c}$. Since $\partial_{-} A_{+}=0$, the integral over $z$ yields for both vertices a $\delta\left(p^{+}-r^{+}\right)$. We note that the above GGR vertex was already obtained in [11]; it differs from the GGR vertex obtained in, e.g., [14,29], which is derived using the direct transition vertex in Fig. 1(a). The above GGR vertex obeys the following important properties: at first one finds current conservation on the level of the vertex, even if the second gluon is not real and/or does not carry physical polarization,

$$
r_{\nu} \cdot \Gamma_{+}^{\nu \mu}(r, p)=0=\Gamma_{+}^{\nu \mu}(r, p) \cdot p_{\mu} .
$$

A disadvantage of the above vertex, already noticed in [11] is that the term $p \cdot r / p^{+}$is in potential conflict with the Steinmann relations [30], since it may yield individual Feynman diagrams which contain singularities in overlapping channels, e.g., the $s$ and the $t$ channels. Nevertheless, since this vertex is obtained from a shift in the gluonic field from an effective action that explicitly obeys the Steinmann relations, the terms that potentially violate the Steinmann relations should cancel for physical quantities. Application of this vertex to the calculation of physical observables should therefore be safe. Apart from the above relation, this GGR vertex also obeys

$$
n_{\nu}^{+} \cdot \Gamma_{+}^{\nu \mu}(r, p)=0=\Gamma_{+}^{\nu \mu}(r, p) \cdot n_{\mu}^{+},
$$

as well as

$$
\Gamma_{+}^{\nu \alpha}(r, k) \cdot\left(-g_{\alpha \alpha^{\prime}}\right) \cdot \Gamma_{+}^{\alpha^{\prime} \mu}(k, p)=-p^{+} \Gamma_{+}^{\nu \mu}(r, p) .
$$

Identical properties hold for the QQR vertex,

$$
\begin{aligned}
\Gamma_{\beta \gamma^{\prime}}(r, p) \not h_{\gamma^{\prime} \gamma} & =0=\not h_{\beta \beta^{\prime}} \Gamma_{\beta^{\prime} \gamma}(r, p), \\
\Gamma_{\beta \gamma}(r, p) \not \not k_{\gamma \gamma^{\prime}} \Gamma_{\gamma^{\prime} \alpha}(r, p) & =-p^{+} \Gamma_{\beta \alpha}(r, p) .
\end{aligned}
$$

\section{Properties of the Reggeized gluon field}

The last two properties, Eqs. (28) and (34), are of high importance to arrive at a summation of the Reggeized gluon field to all orders. Before addressing this task, we first recall the following property of the Reggeized gluon field:

$$
\begin{array}{ll}
\partial_{-} A_{+}(x)=0, & A_{+}(x)=A_{+}\left(x_{0}^{-}, \boldsymbol{x}, x^{+}\right), \\
\partial_{+} A_{-}(x)=0, & A_{-}(x)=A_{-}\left(x_{0}^{+}, \boldsymbol{x}, x^{+}\right),
\end{array}
$$

with a $x_{0}^{ \pm}$as a constant that is common to all $A_{+}$fields; since the scattering amplitude depends by Lorentz invariance not on absolute space-time values, this constant can be conveniently set to $x_{0}^{ \pm}=0$. To keep the presentation as general as possible, we keep in the following, however, the dependence on $x_{0}^{ \pm}$and set it only to zero when comparing to other approaches. We further recall that the propagator of the Reggeized gluon field, Fig. 1(b), which connects clusters significantly separated in rapidity, comes with a purely transverse denominator. The corresponding configuration space propagator is therefore in four dimensions given by

$$
\begin{aligned}
\left\langle A_{+}(x) A_{-}(y)\right\rangle & =\int \frac{d^{4} q}{(2 \pi)^{4}} e^{-i q \cdot(x-y)} \frac{2 i}{\boldsymbol{q}^{2}} \\
= & \frac{1}{2} \int \frac{d^{2} \boldsymbol{q}}{(2 \pi)^{2}} \int \frac{d q^{+}}{2 \pi} e^{-i q^{+}\left(x_{0}^{-}-y^{-}\right) / 2} \\
& \times \int \frac{d q^{-}}{2 \pi} e^{-i q^{-}\left(x^{+}-x_{0}^{+}\right) / 2} e^{i \boldsymbol{q} \cdot(x-y)} \frac{2 i}{\boldsymbol{q}^{2}} \\
= & 4 \delta\left(y^{-}-x_{0}^{-}\right) \delta\left(x^{+}-x_{0}^{+}\right) \cdot \int \frac{d^{2} \boldsymbol{q}}{(2 \pi)^{2}} e^{i \boldsymbol{q} \cdot(x-y)} \frac{i}{\boldsymbol{q}^{2}} .
\end{aligned}
$$


The four-dimensional Reggeized gluon propagator can therefore be interpreted as the propagator of a two-dimensional Reggeized gluon field $\alpha(z)$, together with corresponding delta functions,

$$
\left\langle A_{+}(x) A_{-}(y)\right\rangle=4 \delta\left(x^{+}-x_{0}^{+}\right) \delta\left(y^{-}-x_{0}^{-}\right) \cdot\langle\alpha(\boldsymbol{x}) \alpha(\boldsymbol{y})\rangle,
$$

with

$$
\langle\alpha(\boldsymbol{x}) \alpha(\mathbf{0})\rangle=\int \frac{d^{2} \boldsymbol{q}}{(2 \pi)^{2}} \frac{i e^{i \boldsymbol{q} \cdot(\boldsymbol{x})}}{\boldsymbol{q}^{2}} .
$$

The result then suggests to parametrize the Reggeized gluon field as

$$
A_{+}(x)=2 \cdot \alpha(\boldsymbol{x}) \delta\left(x^{+}-x_{0}^{+}\right)
$$

where the factor of 2 appears due to the chosen convention for light-cone directions. We note that such a parametrization is commonly used in calculations within the CGC formalism (see, e.g., [6-10]) with $x_{0}^{+}=0$. This treatment of the Reggeized gluon field is possible, since the fields $A_{ \pm}$ are within the effective action to be treated as external classical fields for individual rapidity clusters, while they only connect to other clusters through the above Reggeized gluon propagator.

\section{E. All order summation of the Reggeized gluon fields}

To sum up the interaction of partons with Reggeized gluon fields to all orders in $\alpha_{s}$, it is necessary to determine the free gluon propagator of the quantum fluctuations $v^{\mu}$, which requires fixing a gauge following the usual FaddeevPopov procedure. While the following discussion will be based on the covariant gauge, we will also comment on the corresponding results obtained in axial light cone gauge with the free propagators given by the usual expressions

$$
\begin{aligned}
\tilde{G}_{\mathrm{cov}, \mu \nu}^{(0), a b}(k) & =\delta^{a b} \tilde{D}_{0}(k)\left[-g_{\mu \nu}+(1-\xi) \frac{k_{\mu} k_{\nu}}{k^{2}}\right] \\
& =\delta^{a b} d_{\mu \nu}(k, \xi) \tilde{D}_{0}(k), \\
\tilde{G}_{\text {l.c. }, \mu \nu}^{(0), a b}(k) & =\delta^{a b} \tilde{D}_{0}(k)\left[-g_{\mu \nu}+\frac{k_{\mu}\left(n^{+}\right)_{\nu}+\left(n^{+}\right)_{\mu} k_{\nu}}{k \cdot n^{+}}\right] \\
& =\delta^{a b} d_{\text {l.c. }, \mu \nu}\left(k, n^{+}\right) \tilde{D}^{(0)}(k),
\end{aligned}
$$

with

$$
\tilde{D}^{(0)}(k)=\frac{i}{k^{2}+i \epsilon}
$$

If not denoted otherwise, we will in the following always use covariant gauge. For the quark propagator one finds the usual expression

$$
\tilde{S}_{F}^{(0)}(k)=\not k \tilde{D}^{(0)}(k)
$$

Because of the properties Eqs. (26) and (30), connecting two GGR vertices with a gluon propagator, the polarization tensor of the latter reduces always to $-g_{\mu \nu}$, since all other terms are set to zero. Using further the properties Eqs. (28) and (34), the interaction of $n$ Reggeized gluons with a quark or gluon reduces essentially to

$$
\begin{aligned}
& \prod_{i=1}^{n} \int d z_{i}^{4} \prod_{j=1}^{n} \int \frac{d^{4} k_{j}}{(2 \pi)^{4}}\left(-k_{1}^{+}\right) D_{0}\left(k_{1}\right) e^{i k_{1} \cdot\left(z_{1}-z_{2}\right)} \cdots\left(-k_{n-1}^{+}\right) D_{0}\left(k_{n-1}\right) e^{i k_{n-1} \cdot\left(z_{n-1}-z_{n}\right)} \\
& \quad \times e^{-i p \cdot z_{1}}\left(-i g A_{+}\left(z_{n}\right)\right) \cdots\left(-i g A_{+}\left(z_{1}\right)\right) e^{i r \cdot z_{n}} \\
& =-2 \pi \delta\left(p^{+}-r^{+}\right) e^{-i x_{0}^{+}\left(p^{-}-r^{-}\right)} \int d^{2} z e^{i z \cdot(p-r)} \\
& \quad \times\left[\theta\left(p^{+}\right) \mathrm{P}\left(\frac{-g}{2}\right)^{n} \int \prod_{i=1}^{n} d z_{i}^{+} \tilde{A}_{+}\left(z_{i}\right)-\theta\left(-p^{+}\right) \overline{\mathrm{P}}\left(\frac{g}{2}\right)^{n} \int \prod_{i=1}^{n} d z_{i}^{+} \tilde{A}_{+}\left(z_{i}\right)\right]
\end{aligned}
$$

To arrive at the above identity, we used the property Eq. (34). $A_{+}=-i t_{j i}^{c} A_{+}^{c}$ are Reggeized gluon fields in the fundamental representation for quarks while gluons require $A_{+} \rightarrow \tilde{A}_{+}=-i T_{b a}^{c} A_{+}^{c}$, i.e., Reggeized gluon fields in the adjoint representation. (Anti)path ordering of color matrices is defined as

$$
\begin{aligned}
& \mathrm{P} A_{+}\left(z_{n}^{+}, z\right) \cdots A_{+}\left(z_{1}^{+}, z\right) \equiv A_{+}\left(z_{n}^{+}, z\right) \cdots A_{+}\left(z_{1}^{+}, z\right) \theta\left(z_{n}^{+}-z_{n-1}^{+}\right) \cdots \theta\left(z_{2}^{+}-\cdots z_{1}^{+}\right) \\
& \overline{\mathrm{P}} A_{+}\left(z_{n}^{+}, z\right) \cdots A_{+}\left(z_{1}^{+}, z\right) \equiv A_{+}\left(z_{1}^{+}, z\right) \cdots A_{+}\left(z_{n}^{+}, z\right) \theta\left(z_{n}^{+}-z_{n-1}^{+}\right) \cdots \theta\left(z_{2}^{+}-\cdots z_{1}^{+}\right)
\end{aligned}
$$

Summing finally over the number of Reggeized gluons, one obtains for gluons the following effective vertex that sums up the interaction with an arbitrary number of Reggeized gluon fields, 


$$
\begin{aligned}
& \stackrel{p}{\longrightarrow} \underset{\text { ×00 }}{\longrightarrow}=\tau_{G, \nu \mu}^{a b}(p,-r)=-4 \pi \delta\left(p^{+}-r^{+}\right) \Gamma_{\nu \mu}(r, p) e^{-i x_{0}^{+}\left(p^{-}-r^{-}\right)} \\
& \cdot \int d^{2} \boldsymbol{z} e^{i \boldsymbol{z} \cdot(\boldsymbol{p}-\boldsymbol{r})}\left[\theta\left(p^{+}\right)\left[U^{b a}(\boldsymbol{z})-\delta^{a b}\right]-\theta\left(-p^{+}\right)\left[\left[U^{b a}(\boldsymbol{z})\right]^{\dagger}-\delta^{a b}\right]\right] .
\end{aligned}
$$

For quarks one finds

$$
\begin{aligned}
& \longrightarrow \rightarrow{ }^{p}=\tau_{F}(q,-r)=2 \pi \delta\left(p^{+}-r^{+}\right) h^{+} e^{-i x_{0}^{+}\left(p^{-}-r^{-}\right)} \\
& \cdot \int d^{2} \boldsymbol{z} e^{i \boldsymbol{z} \cdot(\boldsymbol{p}-\boldsymbol{r})}\left[\theta\left(p^{+}\right)[W(\boldsymbol{z})-1]-\theta\left(-p^{+}\right)\left[[W(\boldsymbol{z})]^{\dagger}-1\right]\right] .
\end{aligned}
$$

To write down the above expressions, we introduced Wilson lines in the adjoint

$$
\begin{aligned}
U^{a b}(z) & =\mathrm{P} \exp \left(-\frac{g}{2} \int_{-\infty}^{\infty} d z^{+} \tilde{A}_{+}\right), \\
\tilde{A}_{+} & =-i T_{a b}^{c} A_{+}^{c},
\end{aligned}
$$

and the fundamental representation

$$
\begin{aligned}
W(z) & =\mathrm{P} \exp \left(-\frac{g}{2} \int_{-\infty}^{\infty} d z^{+} A_{+}\right), \\
A_{+} & =-i t_{i j}^{c} A_{+}^{c} .
\end{aligned}
$$

In contrast to the notation used in [28,31] and elsewhere, we use here the letter $W$ to denote the Wilson line in the fundamental representation to avoid confusion with the gluonic field in the effective action. The above expressions Eqs. (40) and (41) are one of the central results of this paper.

\section{COMPARISON WITH EXPRESSIONS IN THE LITERATURE}

At this stage it is necessary to compare the result derived from Lipatov's high energy effective action with the conventional quark and gluon propagators in the presence of a background field used in the literature.

\section{A. Comparison with propagators in the presence of a background field}

Corresponding resummed propagators are within the effective action now easily obtained. Using Eqs. (40) and (41) one finds for the resummed quark $\left(S_{F}\right)$ and gluon $(G)$ propagators

$$
\begin{aligned}
S_{F}(p, q)= & S_{F}^{(0)}(p)(2 \pi)^{4} \delta^{(4)}(p-q) \\
& +S_{F}^{(0)}(p) \cdot \tau_{F}(p, q) \cdot S_{F}^{(0)}(q), \\
G_{\mu \nu}^{a d}(p, q)= & G_{\mu \nu}^{(0), a b}(p)(2 \pi)^{4} \delta^{(4)}(p-q) \\
& +G_{\mu \alpha}^{(0), a b}(p) \cdot \tau_{G}^{\alpha \beta, b c}(p, q) \cdot G_{\beta \nu}^{(0), c d}(q),
\end{aligned}
$$

where for the moment we do not specify the gauge of the free gluon propagators. These expressions are now to be compared with propagators obtained from treating the target as a background field in light-cone gauge $b \cdot n_{-}=0$ with the only nonzero component

$$
b_{+}\left(x^{+}, z\right)=\delta\left(x^{+}\right) \beta(z),
$$

while $b_{\perp}^{\mu}=0$. Using the Fourier transform of corresponding counterparts in configuration space (see, e.g., [32]) one finds in momentum space (see, e.g., [31] for expressions used in a recent calculation)

$$
\begin{aligned}
S_{F}^{[b]}(p, q)= & S_{F}^{(0)}(p)(2 \pi)^{4} \delta^{(4)}(p-q) \\
& +S_{F}^{(0)}(p) \cdot \tilde{\tau}_{F}(p, q) \cdot S_{F}^{(0)}(q), \\
G_{\mu \nu}^{[b], a d}(p, q)= & G_{1 . c ., \mu \nu}^{(0), a b}(p)(2 \pi)^{4} \delta^{(4)}(p-q) \\
& +G_{\mu \alpha}^{(0), a b}(p) \cdot \tilde{\tau}_{G}^{\alpha \beta, b c}(p, q) \cdot G_{1 . c ., \beta \nu}^{(0), c d}(q),
\end{aligned}
$$

where the gluon propagator is now restricted to $v \cdot n_{-}=0$ light-cone gauge. The superscript " $[b]$ " indicates that these propagators have been derived using the background field in light-cone gauge and not the Reggeized field $A_{+}$. One has

$$
\begin{aligned}
\tilde{\tau}_{F}(p,-q)= & 2 \pi \delta\left(p^{+}-q^{+}\right) h^{+} \\
& \times \int d^{2} \boldsymbol{z} e^{i z \cdot(\boldsymbol{p}-\boldsymbol{q})}\left\{\theta\left(p^{+}\right)[W[b](\boldsymbol{z})-1]\right. \\
& \left.-\theta\left(-p^{+}\right)\left[W[b]^{\dagger}(\boldsymbol{z})-1\right]\right\}, \\
\tilde{\tau}_{G, \nu \mu}^{a b}(p, q)= & 2 \pi \delta\left(p^{+}-q^{+}\right)\left(-2 p^{+} g_{\nu \mu}\right) \\
& \times \int d^{2} \boldsymbol{z} e^{i \boldsymbol{z} \cdot(\boldsymbol{p}-\boldsymbol{q})}\left\{\theta\left(p^{+}\right)\left[U^{a b}[b](\boldsymbol{z})-1\right]\right. \\
& \left.-\theta\left(-p^{+}\right)\left[\left(U^{a b}[b]\right)^{\dagger}(\boldsymbol{z})-1\right]\right\},
\end{aligned}
$$

with Wilson lines in fundamental $(W)$ and adjoint $(U)$ representation 


$$
\begin{aligned}
W[b](z) & =\mathrm{P} \exp \left(-\frac{g}{2} \int_{-\infty}^{\infty} d x^{+} b^{-, c}\left(x^{+}, z\right) t^{c}\right), \\
b^{-}\left(x^{+}, z\right) & =-i b^{-, c}\left(x^{+}, z\right) t^{c} \\
U[b](z) & =\operatorname{Pexp}\left(-\frac{g}{2} \int_{-\infty}^{\infty} d x^{+} b^{-, c}\left(x^{+}, z\right) T^{c}\right), \\
\tilde{b}^{-}\left(x^{+}, z\right) & =-i b^{-, c}\left(x^{+}, z\right) T^{c} .
\end{aligned}
$$

Leaving aside potential differences in the Wilson lines, to which we will turn in Sec. IV B, one observes that both quark propagators agree directly with each other (if one sets $x_{0}^{+}=0$ ). To carry out a similar comparison for the gluon, we consider first the case where the external free propagators in Eq. (44) are taken in $v \cdot n_{-}=0$ light-cone gauge. Since $d_{1 . c .}^{\mu \nu}\left(p, n^{+}\right) n_{\nu}^{+}=0=d_{1 . c .}^{\mu \nu}\left(r, n^{+}\right) n_{\mu}^{+}$, all terms in the vertex $\Gamma^{\nu \mu}(r, p)$ that contain a $n_{\mu}^{+}$or $n_{\nu}^{+}$vanish. One therefore remains with the $2 p^{+} g_{\mu \nu}$ term only, which is precisely the term used in Eq. (48). Both expressions therefore agree for $x_{0}^{+}=0$. We further note that both the light-cone gauge polarization tensor and the GGR vertex can be factorized into the products of a "left" and a "right" tensor,

$$
\begin{aligned}
& c_{L}^{\mu \alpha}\left(p, n^{+}\right)=\left(g^{\mu \alpha}-\frac{\left(n^{+}\right)^{\mu} p^{\alpha}}{p \cdot n^{+}}\right), \\
& c_{R}^{\alpha \nu}\left(r, n^{+}\right)=\left(g^{\alpha \nu}-\frac{r^{\alpha}\left(n^{+}\right)^{\nu}}{r \cdot n^{+}}\right),
\end{aligned}
$$

where

$$
\Gamma^{\mu \nu}=p^{+} c_{L}^{\mu \alpha}\left(p, n^{+}\right) c_{R}^{\alpha \nu}\left(r, n^{+}\right)
$$

and

$$
d^{\mu \nu}\left(p, n^{+}\right)=c_{R}^{\mu \alpha}\left(p, n^{+}\right)\left(-g_{\alpha \beta}\right) c_{L}^{\beta \nu}\left(p, n^{+}\right) .
$$

This property allows to establish on a diagrammatic level how the vertex $\Gamma^{\mu \nu}$ can build up from properly factorizing the numerator of the light-cone gauge gluon propagator and absorbing them into the vertex; the information contained in Eqs. (44) and (46) is therefore in this sense identical. It is an interesting note aside that a similar mechanism has been used in the construction of a certain projector in [33].

\section{B. Comparison of Wilson lines and the definition of the Reggeized gluon}

In the following we attempt a somewhat detailed comparison between the Wilson lines in the Reggeized gluon field $A_{+}$, arising from Lipatov's high energy effective action, and Wilson lines in the background field $b_{+}$, frequently encountered in CGC calculation in a light-cone gauge. While we find that the interpretation of these Wilson lines differs, we would like to stress that for the calculation of correlators in the dilute quasielastic region, i.e., perturbative forward scattering in the presence of a strong background field (Reggeized gluon or light-cone gauge), both formalisms are equivalent; the only difference is that the effective action allows the use of arbitrary gauges. ${ }^{2}$ The difference therefore lies mainly in the interpretation of the background field, i.e., the coupling to color sources in a different rapidity cluster. At first both Wilson lines appear to resum identical fields; Eqs. (34) and (45) take identical forms. Obviously one has for a Wilson line of a generic gluonic field $V_{+}$,

$$
\begin{aligned}
W[V](x)= & \operatorname{Pexp}\left(-\frac{g}{2} \int_{-\infty}^{\infty} d x^{+} V_{+}(x)\right) \\
= & \sum_{n=0}^{\infty} \frac{(-g)^{n}}{2^{n} n !} \int \prod_{i=1}^{n} d x_{i}^{+} \\
& \times\left[V_{+}\left(x_{1}\right) \cdots V_{+}\left(x_{n}\right) \theta\left(x_{1}^{+}-x_{2}^{+}\right) \cdots \theta\left(x_{n-1}^{+}-x_{n}^{+}\right)\right. \\
& + \text {permutations }] .
\end{aligned}
$$

If now $V_{+}(x)=A_{+}(x)=-2 i \delta\left(x^{+}-x_{0}^{+}\right) \alpha^{a}(\boldsymbol{x}) t^{a}$, the permutations of the fields $A\left(x_{i}\right), i=1, \ldots, n$ are all identical (since their $x^{+}$dependence is identical) and we arrive directly at

$$
\begin{aligned}
W[A](x)= & \sum_{n=0}^{\infty} \frac{1}{n !}\left(\frac{-g}{2}\right)^{n} \prod_{i=1}^{n} \int d x_{i}^{+} A_{+}\left(x_{1}\right) \cdots A_{+}\left(x_{n}\right) \\
& \times\left[\theta\left(x_{1}^{+}-x_{2}^{+}\right) \cdots \theta\left(x_{n-1}^{+}-x_{n}^{+}\right)+\text {permutations }\right] \\
= & \sum_{n=0}^{\infty} \frac{1}{n !}\left(\frac{-g}{2}\right)^{n} \prod_{i=1}^{n} \int d x_{i}^{+} A_{+}\left(x_{1}\right) \cdots A_{+}\left(x_{n}\right) \\
= & e^{i g \alpha^{a}(\boldsymbol{x}) t^{a}} .
\end{aligned}
$$

We therefore obtain a simple matrix exponential. Formally, also the choice $V_{+}(x)=b_{+}(x)=-i \delta\left(x^{+}\right) \beta^{a}\left(\boldsymbol{x}, x^{-}\right) t^{a}$ leads obviously to the same result. In the literature such an interpretation is, however, usually avoided, by treating the contracting of the $x^{+}$dependence to deltalike support as an approximation that applies to the calculation of correlators in the background field, while the $b_{+}$itself is ordered in the $x^{+}$coordinates; see, e.g., [10].

While the precise interpretation used is irrelevant for the calculation of correlators in the presence of a background field, the difference becomes striking once correlators of the background field with, e.g., color charges in a rapidity cluster significantly separated in rapidity are considered ("the dense target"). Vertices that describe the interaction of the Wilson line with $n$-Reggeized gluon fields come with purely symmetric color tensors, since the precise ordering

\footnotetext{
${ }^{2}$ Nevertheless, we stress that calculation based on the background field in the light-cone gauge also allows at least in principle for the use of different gauges for the gluon fluctuations.
} 
of fields is irrelevant. For the gluonic field $b_{+}(x)$ such a result is not acceptable, since one would miss the corresponding antisymmetric and mixed symmetry correlators. Within the effective action, the interaction with these color charges does not occur directly through the Reggeized gluon field, but through the induced vertices of Fig. 1 and corresponding higher order vertices. Following the treatment in [28], these vertices carry only antisymmetric color tensors [corresponding to a combination of anticommutators of $\mathrm{SU}\left(N_{c}\right)$ generators]. Combining these induced vertices with the symmetric $m$ Reggeized gluon state to construct a "Wilson-line- $n$ gluon" vertex $(n \geq m)$, where the coupling to the Wilson line is always mediated by at least one Reggeized gluon, one recovers the complete symmetry structure. For a pedagogic presentation for the case up to three gluons we refer to Appendix A; see also the discussion in [21].

At this point we return to a proposal made in [27] for the definition of the Reggeized gluon from Wilson lines in the Balitsky-JIMWLK formalism. There it has been proposed to define the Reggeized gluon $R^{a}(z)$ as the logarithm of the adjoint Wilson line,

$$
R^{a}(z) \equiv \frac{1}{g N_{c}} f^{a b c} \log U^{b c}(z)
$$

Using the above results, one finds directly for the results obtained from Lipatov's high energy effective action,

$R^{a}(\boldsymbol{z})=\frac{1}{g N_{c}} f^{a b c}\left[i g \alpha^{d}(z) T_{b c}^{d}\right]=\alpha^{a}(\boldsymbol{z})=\frac{1}{2} \int d x^{+} A_{+}^{a}\left(x^{+}, \boldsymbol{z}\right) ;$

i.e., the definition of the Reggeized gluon of [27] coincides with the Reggeized gluon field of Lipatov's effective action, once this field is integrated over the corresponding light-cone coordinate. ${ }^{3}$

\section{BALITSKY-JIMWLK EVOLUTION}

In the following we demonstrate that the high energy evolution of Wilson lines of Reggeized gluons (obtained within the high energy effective action) leads directly to the leading order Balitsky-JIMWLK evolution equation. Even though this is expected, given the coincidence in the resummed gluon and quark propagators, this provides an important consistency check, in particular, for the future calculation of CGC observables. We will then investigate the question whether integrating out quantum fluctuations of a general ensemble of Wilson lines gives indeed rise to the Balitsky-JIMWLK evolution equation.

\footnotetext{
${ }^{3}$ At least within the high energy effective action, a definition based on the Wilson lines in the fundamental representation would be equally possible, i.e., $R^{a}(z)=\frac{2}{i g} \operatorname{tr}\left(t^{a} \log [V(z)]\right)=\alpha^{a}(z)$.
}

Within Lipatov's high energy effective action, the determination of high energy evolution requires in general the high energy effective action for "central-rapidity" processes, i.e., the effective action that contains both $A_{-}$ and $A_{+}$Reggeized gluon fields and corresponding induced vertices. For the discussion of dense-dilute collision the decomposition provided by the effective action for central rapidities is, however, not very efficient; the additional set of induced vertices provides a certain color decomposition of amplitudes that describe gluon production from a multiReggeized gluon exchange. While it has been demonstrated at the level of the scattering amplitude for four-Reggeized gluon exchange that after a certain reshuffling of terms the 2-4 Reggeized gluon vertex (triple Pomeron vertex) arises from the high energy effective action[21] (which at the same time can be shown to arise as well from BalitskyJIMWLK evolution [23]), the calculation is rather cumbersome. While the reformulation of the effective action provided in Sec. III already provides the first simplification, it is easier to recover the Balitsky-JIMWLK evolution equation from the quantum fluctuations of the quasielastic Lagrangian. For an ensemble of Wilson lines the latter are directly proportional to the high energy divergence, without the need to drop any finite terms. We hope to return to the description that uses the high energy effective action for central rapidity processes in a future publication.

For the following discussion it is sufficient to consider Wilson lines in the fundamental representation. While adjoint Wilson lines can be rewritten in terms of fundamental Wilson lines using the well-known relation

$$
U^{a b}(\boldsymbol{z})=2 \operatorname{tr}\left[t^{a} W(\boldsymbol{z}) t^{b} W^{\dagger}(\boldsymbol{z})\right],
$$

the Hermitian conjugate of a fundamental Wilson line follows trivially from the discussion of the fundamental Wilson line. We will therefore consider the quantum fluctuations of an ensemble of $n$ fundamental Wilson lines in the Reggeized gluon fields,

$$
W\left[A_{+}\right]\left(z_{1}\right) \otimes \cdots \otimes W\left[A_{+}\right]\left(z_{n}\right) .
$$

\section{A. Feynman rules for quantum fluctuations of a Wilson line}

Integrating out the quantum fluctuations $v^{\mu}$ is most easily achieved, if one supplements the effective action with an auxiliary complex one-dimensional scalar field, $\varphi=\varphi\left(x^{+}, z, x_{0}^{-}\right)$, where $z, x_{0}^{-}=0$ are constant for the dynamics of the scalar field. The field is charged in the fundamental representation of $S U\left(N_{c}\right)$ and transforms under gauge transformations as

$$
\delta_{L} \varphi=-\chi_{L} \varphi .
$$

The one-dimensional gauge invariant action of this field, which describes interaction with the gluonic field, is given by 


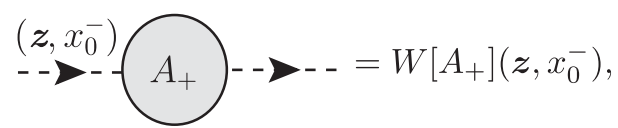

(a)

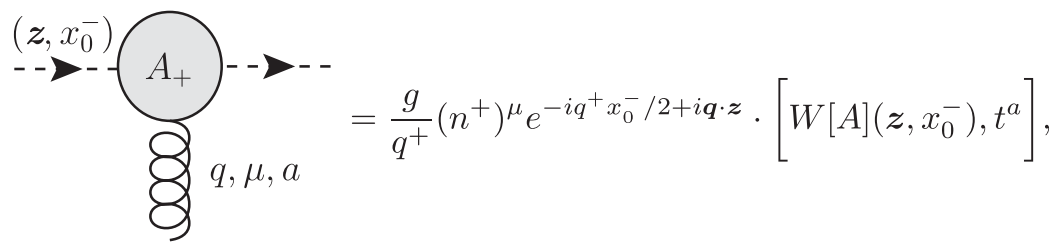

(c)

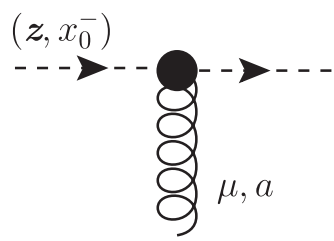

(d)

FIG. 2. Feynman rules for the calculation of quadratic fluctuations of the Wilson lines for covariant or $v_{-}=0$ gauge. Note that the Wilson-line-gluon vertex (d) conserves momentum as usual, while four momenta are not conserved at the vertices (a) and (c). Momenta that are not fixed by external momenta are understood to be integrated over with the measure $d^{4} p /(2 \pi)^{4}$.

$$
S[\varphi, V]=\int d x^{+} \varphi^{\dagger}\left[i \partial_{+}+i g v_{+}\right] \varphi,
$$

where all fields are taken at fixed $\left(\boldsymbol{x}, x_{0}^{-}\right)$. One obtains in a straightforward manner for the propagator of this scalar field

$\left\langle x^{-}\left|\frac{1}{1+\frac{g}{\partial_{+}+\epsilon} V_{+}} \frac{1}{\partial_{+}+\epsilon}\right| y^{-}\right\rangle=\mathrm{P} \exp \left(\frac{-g}{2} \int_{y^{+}}^{x^{+}} d z^{+} v_{+}\right)$.

As a next step we use the parametrization Eq. (17) of the gluonic field and limit ourselves to terms quadratic in the quantum fluctuation. Limiting ourselves further to covariant or $v_{-}=0$ gauges, the following simplified shift is sufficient ${ }^{4}$ : $v^{\mu} \rightarrow V^{\mu}=v^{\mu}+\frac{1}{2}\left(n_{-}\right)^{\mu}\left(A_{+}+\left[A_{+}, \frac{g}{\partial_{-}} v_{-}\right]\right)+\mathcal{O}\left(v_{-}^{2}\right)$.
Expanding our expressions around the background field $g A_{+} \sim 1$, the shifted action is given by

$$
S\left[\varphi, A_{+}, v\right]=\int d x^{+} \varphi^{\dagger}\left[i \partial_{+}+i g\left(v_{+}+A_{+}+\left[A_{+}, \frac{g}{\partial_{-}} v_{-}\right]\right)\right] \varphi .
$$

The resulting set of Feynman rules necessary for the calculation of $\mathcal{O}\left(g^{2}\right)$ corrections within covariant and/or $v_{-}=0$ gauges are then summarized in Fig. 2 .

\section{B. Calculating quantum fluctuations}

Since we require only fluctuations up to quadratic order, it is sufficient to consider the correlator of two Wilson lines at one loop. The nonzero diagrams for self-energy type corrections to one Wilson line are given by

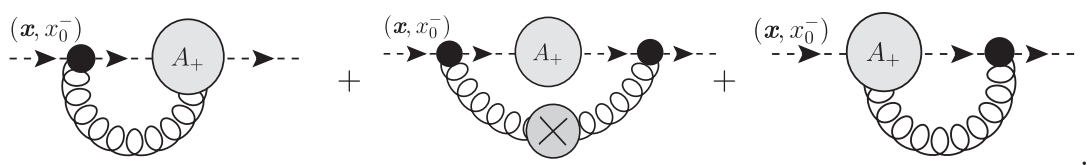

For interactions between two Wilson lines, evaluation of the following diagrams is sufficient (the remaining diagrams can be deduced from symmetry):

\footnotetext{
${ }^{4}$ Covariant gauge requires correlators of $v_{-}$and $v_{+}$fields as well as two $v_{+}$fields; the correlator of two $v_{-}$vanishes on the other hand. $v_{-}=0$ gauge requires on the other hand only the correlator of two $v_{+}$fields.
} 

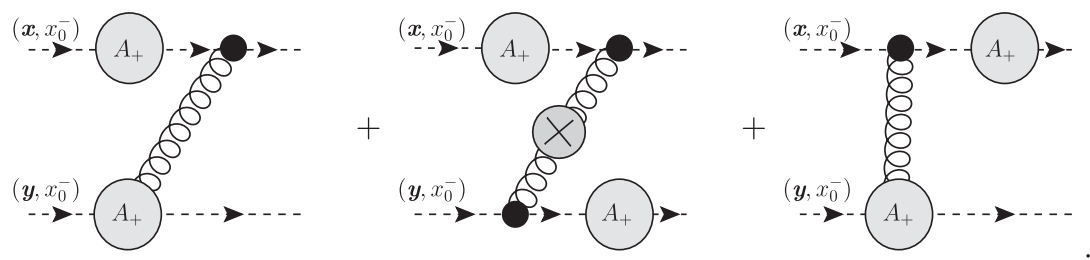

Note that correlators of Wilson lines are only infrared finite, if projected onto the color singlet. The general case of colored Wilson lines is nevertheless of interest; in particular, it allows one to recover the gluon Regge trajectory (see [27] for a detailed discussion). We therefore work in $d=4+2 \epsilon$ space-time dimensions, with the vertices Eqs. (40) and (41) generalizing trivially. We obtain

$$
\begin{gathered}
2 \pi \delta\left(p^{+}-r^{+}\right) \int d^{2+2 \epsilon} \boldsymbol{z} e^{-i \boldsymbol{p} \cdot(\boldsymbol{x}-\boldsymbol{z})} e^{-i \boldsymbol{r} \cdot(\boldsymbol{z}-\boldsymbol{x})} t^{b} V(\boldsymbol{x}) t^{a} \\
\cdot\left[U^{a b}(\boldsymbol{z})-\delta^{a b}\right]-\theta\left(-p^{+}\right)\left[\left[U^{a b}(\boldsymbol{z})\right]^{\dagger}-\delta^{a b}\right] \\
=\frac{g^{2}}{\pi} \int_{0}^{\infty} \frac{d p^{d}}{p^{+}} \int d^{2+2 \epsilon} \boldsymbol{z} t^{b} V(\boldsymbol{x}) t^{a}\left[U^{a b}(\boldsymbol{z})-\delta^{a b}\right] \frac{\Gamma^{2}(1+\epsilon)}{\left(4 \pi^{2+2 \epsilon}\right)} \frac{-i}{\left([\boldsymbol{x}-\boldsymbol{z})^{2}\right]^{1+\epsilon}\left[(\boldsymbol{x}-\boldsymbol{z})^{2}\right]^{1+\epsilon}}
\end{gathered}
$$

The divergent integral over the plus momenta provides the high-energy singularity that defines the kernel of the high energy evolution. The precise choice of the regulator is irrelevant for leading order accuracy. In the following we chose $\Lambda_{a, b} \rightarrow \infty$ and a scale $s_{0}$ of the order of the transverse scale, also known as the Reggeization scale, to regularize the integral as

$$
\int_{s_{0} / \Lambda^{b}}^{\Lambda^{a}} \frac{d p^{+}}{p^{+}}=\ln \left(\frac{\Lambda_{a} \Lambda_{b}}{s_{0}}\right) .
$$

To derive the high energy evolution of Wilson lines, $\Lambda_{a}$ will be the regulator of interest, since it limits the $p^{+}$integral from above. With the $\overline{\mathrm{MS}}$ strong coupling constant in $d=4+2 \epsilon$ dimensions

$$
\alpha_{s}=\frac{g^{2} \mu^{2 \epsilon} \Gamma(1-\epsilon)}{(4 \pi)^{1+\epsilon}},
$$

we obtain

$$
\begin{aligned}
& \text { e }(x)^{9}=\ln \left(\frac{\Lambda_{a} \Lambda_{b}}{s_{0}}\right) \frac{\alpha_{s}}{\pi^{2}}\left(\frac{4}{\pi \mu^{2}}\right)^{\epsilon} \frac{\Gamma(1+\epsilon)^{2}}{\Gamma(1-\epsilon)} \\
& \qquad d^{2+2 \epsilon} \boldsymbol{z} \frac{(\boldsymbol{x}-\boldsymbol{z}) \cdot(\boldsymbol{x}-\boldsymbol{z})}{\left[(\boldsymbol{x}-\boldsymbol{z})^{2}\right]^{1+\epsilon}\left[(\boldsymbol{x}-\boldsymbol{z})^{2}\right]^{1+\epsilon}} t^{b} W(\boldsymbol{x}) t^{a}\left[U^{b a}(\boldsymbol{z})-\delta^{a b}\right] .
\end{aligned}
$$

We further have

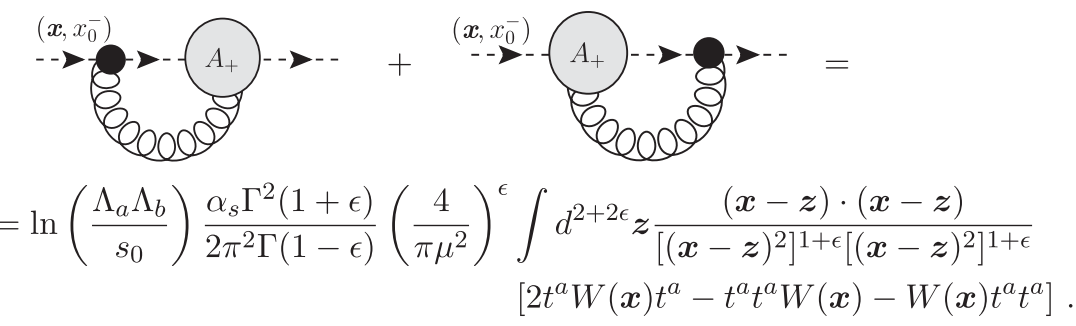


Combining both contributions one obtains

$$
\ln \left(\frac{\Lambda_{a} \Lambda_{b}}{s_{0}}\right) \frac{\alpha_{s} \Gamma^{2}(1+\epsilon)}{2 \pi^{2} \Gamma(1-\epsilon)}\left(\frac{4}{\pi \mu^{2}}\right)^{\epsilon} \int d^{2+2 \epsilon} z \frac{(\boldsymbol{x}-\boldsymbol{z}) \cdot(\boldsymbol{x}-\boldsymbol{z})}{\left[(\boldsymbol{x}-\boldsymbol{z})^{2}\right]^{1+\epsilon}\left[(\boldsymbol{x}-\boldsymbol{z})^{2}\right]^{1+\epsilon}}\left[2 U^{b a}(\boldsymbol{z}) t^{b} W(\boldsymbol{x}) t^{a}-t^{a} t^{a} W(\boldsymbol{x})-W(\boldsymbol{x}) t^{a} t^{a}\right] .
$$

The calculation for the interaction of two Wilson lines follows in complete analogy:

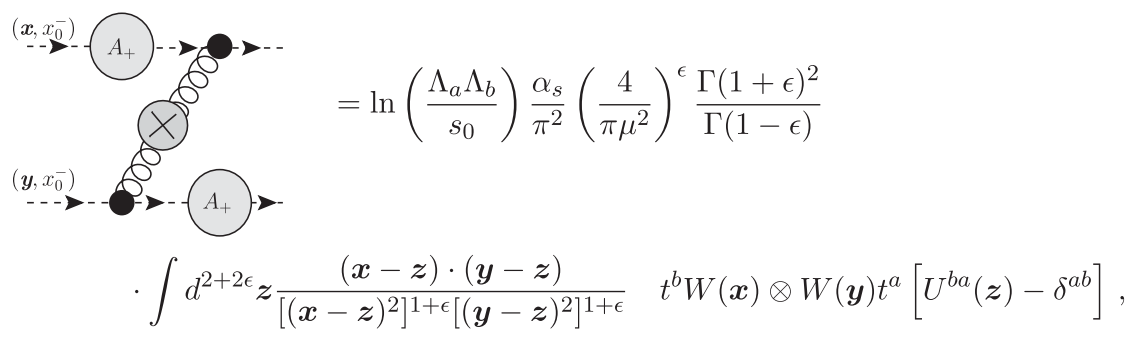

and

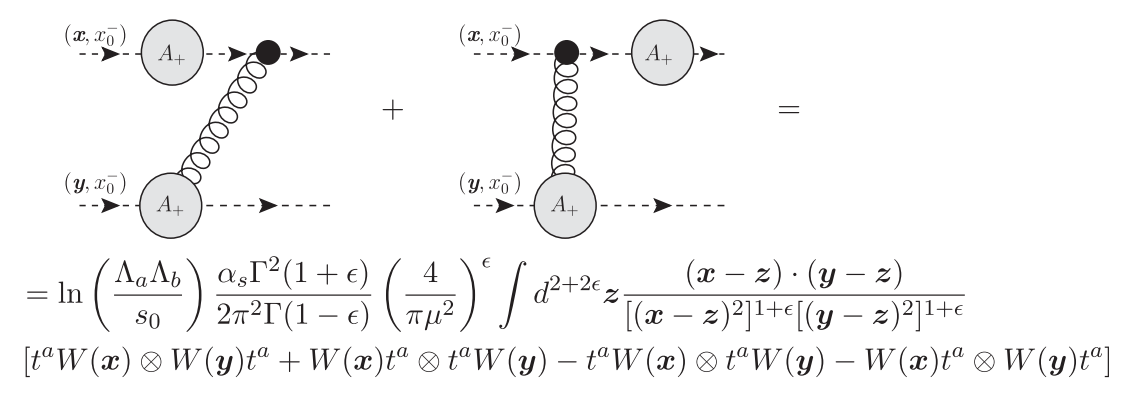

We then obtain for the complete correlator of two Wilson lines

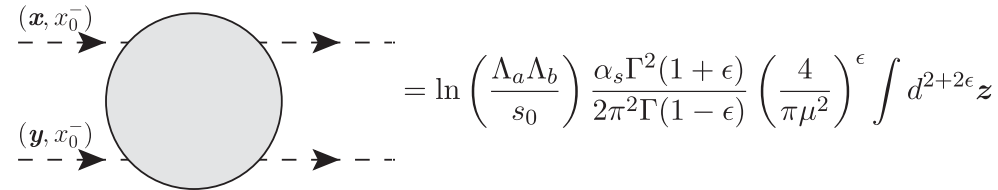

$$
\begin{aligned}
& \left\{\frac{(\boldsymbol{x}-\boldsymbol{z}) \cdot(\boldsymbol{x}-\boldsymbol{z})}{\left[(\boldsymbol{x}-\boldsymbol{z})^{2}\right]^{1+\epsilon}\left[(\boldsymbol{x}-\boldsymbol{z})^{2}\right]^{1+\epsilon}}\left[2 U^{a b}(\boldsymbol{z}) t^{b} W(\boldsymbol{x}) t^{a}-t^{a} t^{a} W(\boldsymbol{x})-W(\boldsymbol{x}) t^{a} t^{a}\right] \otimes W(\boldsymbol{y})\right. \\
& +\frac{(\boldsymbol{y}-\boldsymbol{z}) \cdot(\boldsymbol{y}-\boldsymbol{z})}{\left[(\boldsymbol{y}-\boldsymbol{z})^{2}\right]^{1+\epsilon}\left[(\boldsymbol{y}-\boldsymbol{z})^{2}\right]^{1+\epsilon}}\left[2 U^{a b}(\boldsymbol{z}) t^{b} W(\boldsymbol{y}) t^{a}-t^{a} t^{a} W(\boldsymbol{y})-W(\boldsymbol{y}) t^{a} t^{a}\right] \otimes W(\boldsymbol{x}) \\
& +\frac{(\boldsymbol{x}-\boldsymbol{z}) \cdot(\boldsymbol{y}-\boldsymbol{z})}{\left[(\boldsymbol{x}-\boldsymbol{z})^{2}\right]^{1+\epsilon}\left[(\boldsymbol{y}-\boldsymbol{z})^{2}\right]^{1+\epsilon}}\left[-2 t^{a} W(\boldsymbol{x}) \otimes t^{a} W(\boldsymbol{y})-2 W(\boldsymbol{x}) t^{a} \otimes W(\boldsymbol{y}) t^{a}\right. \\
& \left.\left.+2 U^{a b}(\boldsymbol{z}) t^{a} W(\boldsymbol{x}) \otimes W(\boldsymbol{y}) t^{b}+2 U^{a b}(\boldsymbol{z}) t^{a} W(\boldsymbol{y}) \otimes W(\boldsymbol{x}) t^{b}\right]\right\} .
\end{aligned}
$$

Using the above result it is straightforward to obtain the high energy evolution of an ensemble of $n$ Wilson lines as

$$
-\Lambda_{a} \frac{d}{d \Lambda_{a}}\left[W\left(\boldsymbol{x}_{1}\right) \otimes \cdots \otimes W\left(\boldsymbol{x}_{n}\right)\right]=\sum_{i, j=1} H_{i j}\left[W\left(\boldsymbol{x}_{1}\right) \otimes \cdots \otimes W\left(\boldsymbol{x}_{n}\right)\right],
$$

with the Balitsky-JIMWLK Hamiltonian

$$
\begin{aligned}
H_{i j}= & \frac{\alpha_{s} \Gamma^{2}(1+\epsilon)}{2 \pi^{2} \Gamma(1-\epsilon)}\left(\frac{4}{\pi \mu^{2}}\right)^{\epsilon} \int d^{2+2 \epsilon} z \frac{\left(\boldsymbol{x}_{i}-z\right) \cdot\left(\boldsymbol{x}_{j}-z\right)}{\left[\left(\boldsymbol{x}_{i}-\boldsymbol{z}\right)^{2}\right]^{1+\epsilon}\left[\left(\boldsymbol{x}_{j}-\boldsymbol{z}\right)^{2}\right]^{1+\epsilon}} \\
& \times\left[T_{i, L}^{a} T_{j, L}^{a}+T_{i, R}^{a} T_{j, R}^{a}-U^{a b}(\boldsymbol{z})\left(T_{i, L}^{a} T_{j, R}^{b}+T_{j, L}^{a} T_{i, R}^{b}\right)\right] .
\end{aligned}
$$


In the presentation we followed here closely [27] and define $T_{L, i}^{a}$ and $T_{R, j}^{a}$ as the group generators acting to the left (L) or to the right (R) on the Wilson line $W\left(\boldsymbol{x}_{i}\right)$,

$$
T_{L, i}^{a}\left[W\left(z_{i}\right)\right] \equiv t^{a} W\left(z_{i}\right), \quad T_{R, i}^{a}\left[W\left(z_{i}\right)\right] \equiv W\left(z_{i}\right) t^{a} .
$$

\section{CONCLUSION AND OUTLOOK}

We investigated to which extent it is possible to obtain within Lipatov's high energy effective action gluon and quark propagators, which resum interaction with a strong (Reggeized) gluon background field, and whether the effective action allows one to rederive Balitsky-JIMWLK evolution. We found that both questions can be answered positively. To arrive at this result, we used a special parametrization of the gluonic field, already proposed in [11]. This parametrization both allows an expansion of the gluonic field around the Reggeized gluon field - which is assumed to be strong — and provides consistent gauge transformation properties for the parametrized gluonic field. Expanding the resulting effective Lagrangian up to quadratic order in quantum fluctuations around the strong Reggeized gluon field, we obtain a new kind of gluon-gluon-Reggeized gluon vertex as well as the usually quark-quark-Reggeized gluon vertex. Both vertices allow for a straightforward resummation of the Reggeized gluon field to all orders into Wilson lines. The resulting resummed gluon and quark propagators agree for $v_{-}=0$ light-cone gauge with corresponding propagators that include all order resummation of a gluonic background field in lightcone gauge. The latter is frequently employed in the calculation of perturbative observables in the presence of high parton densities, in particular, within the color glass condensate effective theory. Finally we demonstrated that these propagators allow us to recover the complete (leading order) Balitsky-JIMWLK evolution equation for Wilson lines from Lipatov's high energy effective action.

Our results demonstrate that high energy factorization as formulated within the Balitsky-JIMWLK evolution and high energy factorization as formulated within Lipatov's high energy effective action are equivalent. At the same time, Lipatov's high energy effective action provides additional flexibility for actual calculations, since it allows one to adopt in a straightforward manner different gauges to determine quantum fluctuations of the gluonic field. Moreover, a matching of results obtained within the BFKL formalism and Lipatov's high energy effective action on the one hand and light-front perturbation theory and the color glass condensate should now be facilitated. As an important side result we confirm the proposed determination of the Reggeized gluon from Balitsky-JIMWLK evolution proposed in [27], within the context of Lipatov's high energy effective action.

Future lines of research need to address the mentioned matching of NLO results obtained within the two different frameworks as well as the explicit calculation of new NLO observables. Even though a number of important NLO results have been obtained in the past for the scattering of a perturbative projectile on a dense target (see, e.g., [34]), there is still a need to refine the available tools for such calculations. Another direction of research needs to address the possible description of central production processes at high parton densities as required for the analysis of nucleus-nucleus collisions and/or high multiplicity events. While the current study is limited to the quasielastic region, such a program requires the investigation of the corresponding effective action that contains induced terms for both plus and minus Reggeized gluon fields. This is also related to the question whether such central production terms can be formulated in a way that automatically gives rise to the Balitsky-JIMWLK hierarchy. Related to this question is the possible extension of the Balitsky-JIMWLK evolution to exclusive observables, generalizing already existing results [35].

\section{ACKNOWLEDGMENTS}

Conversations with Lev N. Lipatov, Jochen Bartels, Agustin Sabio Vera, Grigorios Chachamis, Jose D. Madrigal Martinez, and Krzysztof Kutak on the effective action and related topics as well as collaboration with Alejandra Ayala, Jamal Jalilian-Marian, and Maria-Elena Tejeda Yeomans at an early stage of this project are gratefully acknowledged.

\section{APPENDIX A: MULTIGLUON EXCHANGE WITHIN THE HIGH ENERGY EFFECTIVE ACTION}

We consider in the following the interaction of a Wilson line in the fundamental representation with a color current, where the interaction is mediated through the exchange of Reggeized gluons. To embed the Wilson line into a physical process (and to take the regarding high energy limit), one can, for instance, use the vertex Eq. (41), and combine it with corresponding quark spinors; this relates then to the following discussion of scattering of a quark on a color current. For definiteness we take for the color current on which the Wilson is scattering a quark. The following result does not depend on those details. We are further only interested in $t$-channel gluon exchange of (high energy) gluons between the Wilson line and the color current; couplings of the Reggeized gluon to the quark therefore take place through the QCD quark-gluon vertex as well as induced vertices Fig. 1.

Starting with two gluon exchange as the first nontrivial contribution we have within conventional QCD the two diagrams depicted in Figs. 3(a) and 3(b), while the two relevant contributions within the high energy effective action are given in Figs. 3(c)-3(e). The black blob denotes the various couplings of the Reggeized gluon to the Wilson line. For two Reggeized gluons one has 


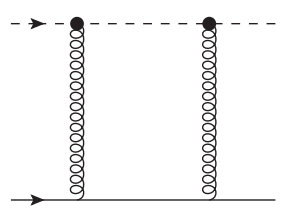

(a)

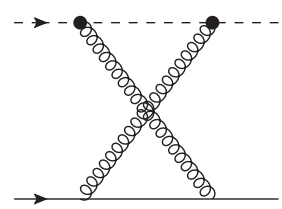

(b)

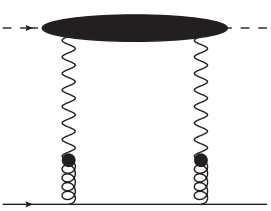

(c)

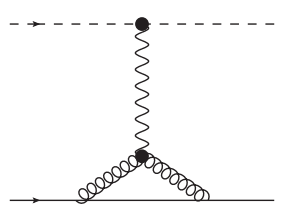

(d)

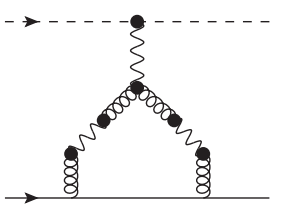

(e)

FIG. 3. Left: Two gluon exchange within QCD. Right: The corresponding decomposition within the high energy effective action in symmetric (two Reggeized gluon exchange) and antisymmetric contributions.

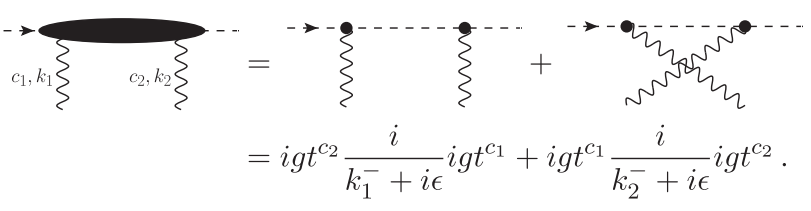

(A1)

Because of high-energy kinematics, the loop integral in the diagram with two Reggeized gluon exchanges of Fig. 3(c) factorizes. It is therefore possible to associate the integration over minus momentum directly with the Wilson line,

$$
\begin{aligned}
\left.\left.\int \frac{d k_{1}^{-}}{2 \pi} \int \frac{d k_{2}^{-}}{2 \pi} 2 \pi \delta\left(k_{1}^{-}+k_{2}^{-}\right){ }_{c_{1}, k_{1}}\right\} \quad c_{c_{2}, k_{2}}\right\} & =(i g)^{2} \cdot \frac{1}{2}\left(t^{c_{1}} t^{c_{2}}+t^{c_{2}} t^{c_{1}}\right) \\
& =(i g)^{2} S_{2}(12) .
\end{aligned}
$$

In the above we used a shorthand notation, introduced in [28],

$$
[i, j] \equiv\left[t^{c_{i}}, t^{c_{j}}\right], \quad S_{n}(1 \cdots n) \equiv \frac{1}{n !} \sum_{i_{1}, \ldots, i_{n}} t^{c_{i_{1}} \cdots t^{c_{i_{n}}}}
$$

where in the second term the sum is taken over all permutations of the numbers $1, \ldots, n$. Using this notation, a possible decomposition of a color tensor with two adjoint color indices is given by the following basis:

$$
[1,2], \quad S_{2}(12) \text {. }
$$

In [28], this decomposition has been used to construct the pole prescription for induced vertices, by projecting out the antisymmetric sector of the complete color structure of a Wilson line. Using this pole prescription and associating the integration over minus momentum similar with the one Reggeized gluon to the two Reggeized gluon splitting, similar to Eq. (A2), it is then straightforward to demonstrate that diagrams such as Fig. 3(e) vanish. We note that this holds for all splittings of a single Reggeized gluons into $n$ Reggeized gluons at tree level; i.e., such splittings are generally absent within this particular pole prescription ${ }^{5}$ after integration over corresponding light-cone momenta. The only diagrams left are therefore Figs. 3(c) and 3(d), where the induced vertex associated with Fig. 3(d) carries the color tensor [1,2], providing therefore the antisymmetric contribution missing in Eq. (A2). For an explicit decomposition of diagrams such as Figs. 3(a) and 3(b), we refer the interested reader to $[21,28]$.

The corresponding symmetry decomposition for three adjoint color indices is provided by the following six tensors:

$$
\begin{array}{lrr}
{[[3,1], 2],} & {[[3,2], 1],} & S_{2}([1,2] 3), \\
S_{2}([1,3] 2), & S_{2}([2,3] 1), & S_{3}(123),
\end{array}
$$

with

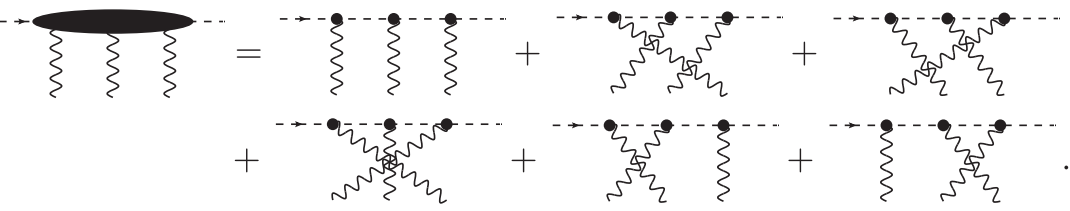

It is straightforward to demonstrate that

\footnotetext{
${ }^{5}$ We note that a prescription different from the one of [28] has been used in [20]. We point out the possibility that the arguments presented here may not hold for this particular prescription.
} 


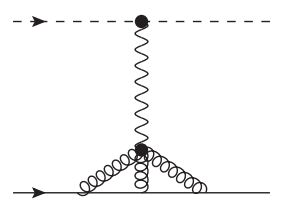

(a)

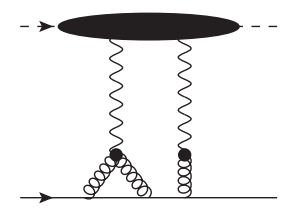

(b)

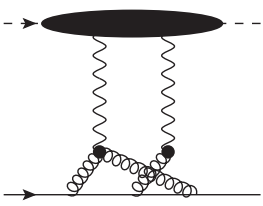

(c)

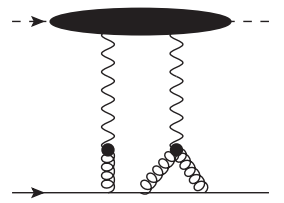

(d)

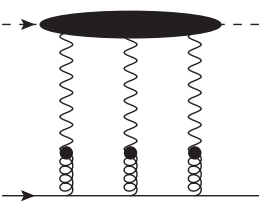

(e)

FIG. 4. Three gluon exchange within the high energy effective action. Left: The antisymmetric contribution. Center: The contribution with mixed symmetry. Right: The symmetric contribution.

$$
\int \frac{d k_{1}^{-}}{2 \pi} \int \frac{d k_{2}^{-}}{2 \pi} \int \frac{d k_{3}^{-}}{2 \pi} 2 \pi \delta\left(k_{1}^{-}+k_{2}^{-}+k_{3}^{-}\right) \sum_{\xi}^{-\cdots}=(i g)^{3} S_{3}(123) .
$$

We therefore find that Fig. 4(a) represents the color tensor $[[3,1], 2]$ and $[[3,2], 1]$ through the color tensors contained in the induced vertex Fig. 1. Figures 4(b)-4(d) provide the color tensors $S_{2}([1,2] 3), S_{2}([1,3] 2)$, and $S_{2}([2,3] 1)$, through the combination of the symmetric two Reggeized gluon state with the induced vertex. Finally Fig. 4(e) provides the color tensor $S_{3}(123)$. For the explicit construction of the Wilson line with three gluons decomposed into the above color tensors, we again refer the interested reader to [21,28], where furthermore some details on the four gluon exchange can be found. The general picture should be nevertheless already clear at this stage: even though the color tensor associated with $n$ Reggeized gluons coupled to a Wilson line is automatically symmetric, the high energy effective action is capable to construct the complete color structure provided by path ordered gluons making use of the additional induced vertices. The latter provides the necessary antisymmetric color tensors as well as corresponding terms of mixed symmetry if combined with multiple Reggeized gluon exchange.

\section{APPENDIX B: QUANTUM FLUCTUATIONS OF WILSON LINE}

In the following we provide further details on the derivation of the Feynman rules for the calculation of Wilson lines. The propagator without fluctuations is easily obtained from the action Eq. (63). In particular,

$$
\begin{aligned}
& \left\langle\infty\left|\frac{1}{1+\frac{g}{\partial_{+}+\epsilon} A_{+}} \frac{1}{\partial_{+}+\epsilon}\right|-\infty\right\rangle \\
& \quad=\operatorname{Pexp}\left(\frac{-g}{2} \int_{-\infty}^{\infty} d z^{+} A_{+}\right)=W\left[A_{+}\right]\left(z, x_{0}^{-}\right) .
\end{aligned}
$$

To include fluctuations, we first consider the case $A_{+} \rightarrow A_{+}+\left[A_{+}, \frac{g}{\partial_{-}} v_{-}\right]$. Since $\partial_{-} A_{+}=0$, the operator $1 / \partial_{-}$does not act on the $A_{+}$fields. We therefore consider a shift of the form $A_{+}(x) \rightarrow A_{+}(x)+\left[A_{+}(x), w(x)\right]=$ $A_{+}(x)+\left[A_{+}(x), w\left(0, \boldsymbol{x}, x^{-}\right)\right]$with $w(x)=\frac{g}{\partial_{-}} v_{-}(x)$ and where we used the delta function implicitly contained in $A_{+}$to set $x^{+}=0$ in the fluctuation $w(x)$. Expanding to linear order in $w$,

$$
\begin{aligned}
\left(A_{+}+\left[A_{+}(x), w(x)\right]\right)^{n} & =A_{+}^{n}+\sum_{i=0}^{n-1}\left(A_{+}^{i+1} w A_{+}^{n-i-1}-A_{+}^{i} w A_{+}^{n-i}\right)+\mathcal{O}\left(w^{2}\right)=A_{+}^{n}+\sum_{i=1}^{n} A_{+}^{i} w A_{+}^{n-i}-\sum_{i=0}^{n-1} A_{+}^{i} w A_{+}^{n-i}+\mathcal{O}\left(w^{2}\right) \\
& =A_{+}^{n}+A_{+}^{n} w-w A_{+}^{n}+\mathcal{O}\left(w^{2}\right)
\end{aligned}
$$

one finds

$$
W\left[A^{+}+\left[A^{+}, w\right]\right]\left(\boldsymbol{x}, x^{-}\right)=W\left[A^{+}\right]+W\left[A^{+}\right] \cdot w(x)-w(x) \cdot W\left[A^{+}\right]+\mathcal{O}\left(w^{2}\right),
$$

where $w(x)=w\left(x^{+}=0, \boldsymbol{x}, x^{-}\right)$. The fluctuation $A_{+} \rightarrow A_{+}+\left[A_{+}, \frac{g}{\partial_{-}} v_{-}\right]$leads therefore to

$$
\frac{g}{\partial_{-}}\left[W\left[A_{+}\right]\left(\boldsymbol{x}, x_{0}^{-}\right), v_{-}(x)\right]=\frac{g}{2} \int_{-\infty}^{x_{0}^{-}} d x^{-}\left[W\left[A_{+}\right]\left(\boldsymbol{x}, x_{0}^{-}\right), v_{-}(x)\right],
$$

which translates directly into the Feynman rule of Fig. 2(c). The second type of fluctuations requires a shift of the form

$$
V_{+}(x)=A_{+}(x)+v_{+}(x),
$$

where $v_{+}$does not have deltalike support. One finds to linear order in the fluctuations $v_{+}(x)$, 


$$
\begin{aligned}
\left.W[A+v](x)\right|_{x^{+}=\infty}= & \left.W[A](x)\right|_{x^{+}=\infty}+\sum_{n=0}^{\infty}\left(\frac{-g}{2}\right)^{n} \prod_{i=1}^{n} \int d x_{i}^{+} \sum_{j=1}^{n} A_{+}\left(x_{1}\right) \cdots A_{+}\left(x_{j-1}\right) v_{+}\left(x_{j}\right) A_{+}\left(x_{j+1}\right) \cdots A_{+}\left(x_{n}\right) \\
& \times \theta\left(x_{1}^{+}-x_{2}^{+}\right) \cdots \theta\left(x_{j-1}^{+}-x_{j}^{+}\right) \theta\left(x_{j}^{+}-x_{j+1}^{+}\right) \cdots \theta\left(x_{n-1}^{+}-x_{n}^{+}\right)+\mathcal{O}\left(v_{+}^{2}\right) .
\end{aligned}
$$

$A_{+}(x) \sim \delta\left(x^{+}-x_{0}^{+}\right)$sets now $\theta\left(x_{j-1}^{+}-x_{j}^{+}\right) \theta\left(x_{j}^{+}-x_{j+1}^{+}\right) \rightarrow \theta\left(x_{0}^{+}-x_{j}^{+}\right) \theta\left(x_{j}^{+}-x_{0}^{+}\right)$. The integral over $x_{j}^{+}$has therefore zero support and yields zero result. The only contributions that remain are $j=1$ and $j=n$, i.e., the cases where the $v_{+}$is placed as the first or the last term. For a term with $m$ fluctuations one therefore finds

$$
\begin{aligned}
& \left(\frac{-g}{2}\right)^{m} \sum_{n=0}^{m} \prod_{i=1}^{n} \prod_{j=n+1}^{m} \int_{x_{0}^{+}}^{\infty} d x_{i}^{+} \int_{-\infty}^{x_{0}^{+}} d x_{j}^{+} v_{+}\left(x_{1}\right) \cdots v_{+}\left(x_{n}\right) W\left[A_{+}\right] v_{+}\left(x_{n+1}\right) \cdots v_{+}\left(x_{m}\right) \\
& \quad \times \theta\left(x_{1}^{+}-x_{2}^{+}\right) \cdots \theta\left(x_{n-1}^{+}-x_{n}^{+}\right) \theta\left(x_{n+1}^{+}-x_{n+1}^{+}\right) \cdots \theta\left(x_{m-1}^{+}-x_{m}^{+}\right) .
\end{aligned}
$$

Fluctuations $A_{+} \rightarrow A_{+}+v_{+}$are therefore taken into account through a Wilson line gluon vertex (see Fig. 2), which can be inserted only before or after the $A_{+}$fields to the Wilson line.

[1] V. S. Fadin, E. A. Kuraev, and L. N. Lipatov, Phys. Lett. 60B, 50 (1975); Zh. Eksp. Teor. Fiz. 71, 840 (1976) [Sov. Phys. JETP 44, 443 (1976)]; Zh. Eksp. Teor. Fiz. 72, 377 (1977) [Sov. Phys. JETP 45, 199 (1977)].

[2] I. I. Balitsky and L. N. Lipatov, Sov. J. Nucl. Phys. 28, 822 (1978).

[3] V. S. Fadin and L. N. Lipatov, Phys. Lett. B 429, 127 (1998); M. Ciafaloni and G. Camici, Phys. Lett. B 430, 349 (1998).

[4] M. Hentschinski, A. Sabio Vera, and C. Salas, Phys. Rev. Lett. 110, 041601 (2013); Phys. Rev. D 87, 076005 (2013); I. Bautista, A. Fernandez Tellez, and M. Hentschinski, Phys. Rev. D 94, 054002 (2016).

[5] F. Gelis, E. Iancu, J. Jalilian-Marian, and R. Venugopalan, Annu. Rev. Nucl. Part. Sci. 60, 463 (2010).

[6] I. Balitsky, Nucl. Phys. B463, 99 (1996).

[7] J. Jalilian-Marian, A. Kovner, L.D. McLerran, and H. Weigert, Phys. Rev. D 55, 5414 (1997); J. JalilianMarian, A. Kovner, A. Leonidov, and H. Weigert, Phys. Rev. D 59, 014014 (1998); 59, 034007 (1999); J. Jalilian-Marian, A. Kovner, and H. Weigert, Phys. Rev. D 59, 014015 (1998).

[8] J. Jalilian-Marian, A. Kovner, A. Leonidov, and H. Weigert, Nucl. Phys. B504, 415 (1997).

[9] A. Kovner, J. G. Milhano, and H. Weigert, Phys. Rev. D 62, 114005 (2000); A. Kovner and J. G. Milhano, Phys. Rev. D 61, 014012 (1999).

[10] E. Iancu, A. Leonidov, and L. D. McLerran, Nucl. Phys. A692, 583 (2001); Phys. Lett. B 510, 133 (2001); E. Ferreiro, E. Iancu, A. Leonidov, and L. McLerran, Nucl. Phys. A703, 489 (2002).

[11] L. N. Lipatov, Nucl. Phys. B452, 369 (1995).

[12] L. N. Lipatov, Phys. Rep. 286, 131 (1997).

[13] M. Hentschinski and A. Sabio Vera, Phys. Rev. D 85, 056006 (2012).
[14] G. Chachamis, M. Hentschinski, J.D. Madrigal, and A. Sabio Vera, Phys. Rev. D 87, 076009 (2013).

[15] M. Hentschinski, J. D. M. Martínez, B. Murdaca, and A. Sabio Vera, Nucl. Phys. B889, 549 (2014); B887, 309 (2014); Phys. Lett. B 735, 168 (2014).

[16] G. Chachamis, M. Hentschinski, J.D. Madrigal, and A. Sabio Vera, Nucl. Phys. B876, 453 (2013); B861, 133 (2012).

[17] J. Bartels, V. S. Fadin, L. N. Lipatov, and G. P. Vacca, Nucl. Phys. B867, 827 (2013).

[18] G. Chachamis, M. Hentschinski, J.D. Madrigal, and A. Sabio Vera, Phys. Part. Nucl. 45, 788 (2014).

[19] M. Nefedov and V. Saleev, Mod. Phys. Lett. A 32, 1750207 (2017).

[20] M. A. Braun and M. Y. Salykin, Eur. Phys. J. C 77, 498 (2017); M. A. Braun, S. S. Pozdnyakov, M. Y. Salykin, and M. I. Vyazovsky, Eur. Phys. J. C 73, 2572 (2013) [Bull. Russ. Acad. Sci. Phys. 80, 959 (2016)]; M. A. Braun and M. I. Vyazovsky, Phys. Rev. D 93, 065026 (2016); M. A. Braun, S. S. Pozdnyakov, M. Y. Salykin, and M. I. Vyazovsky, Eur. Phys. J. C 75, 222 (2015); M. A. Braun, S. S. Pozdnyakov, M. Y. Salykin, and M. I. Vyazovsky, Eur. Phys. J. C 74, 2989 (2014); M. A. Braun, L. N. Lipatov, M. Y. Salykin, and M. I. Vyazovsky, Eur. Phys. J. C 71, 1639 (2011).

[21] M. Hentschinski, arXiv:0908.2576.

[22] M. Hentschinski, Nucl. Phys. B, Proc. Suppl. 198, 108 (2010).

[23] J. Bartels, L. N. Lipatov, and G. P. Vacca, Nucl. Phys. B706, 391 (2005); G. A. Chirilli, L. Szymanowski, and S. Wallon, Phys. Rev. D 83, 014020 (2011).

[24] A. Ayala, E. R. Cazaroto, L. A. Hernández, J. JalilianMarian, and M.E. Tejeda-Yeomans, Phys. Rev. D 90, 074037 (2014); J. Jalilian-Marian, Phys. Rev. D 85, 014037 (2012). 
[25] Y. Hatta, E. Iancu, L. McLerran, A. Stasto, and D. N. Triantafyllopoulos, Nucl. Phys. A764, 423 (2006); Y. Hatta, Nucl. Phys. A781, 104 (2007).

[26] S. Bondarenko and M. A. Zubkov, arXiv:1801.08066; S. Bondarenko, L. Lipatov, S. Pozdnyakov, and A. Prygarin, Eur. Phys. J. C 77, 630 (2017); 77, 527 (2017).

[27] S. Caron-Huot, J. High Energy Phys. 05 (2015) 093.

[28] M. Hentschinski, Nucl. Phys. B859, 129 (2012).

[29] E. N. Antonov, L. N. Lipatov, E. A. Kuraev, and I. O. Cherednikov, Nucl. Phys. B721, 111 (2005).

[30] O. Steinmann, Helv. Phys. Acta 33, 257 (1960); 33, 298 (1960).

[31] A. Ayala, M. Hentschinski, J. Jalilian-Marian, and M. E. Tejeda-Yeomans, Nucl. Phys. B920, 232 (2017); Phys. Lett. B 761, 229 (2016).

[32] L. D. McLerran and R. Venugopalan, Phys. Rev. D 50, 2225 (1994); A. J. Baltz, F. Gelis, L. D. McLerran, and A. Peshier,
Nucl. Phys. A695, 395 (2001); F. Gelis and A. Peshier, Nucl. Phys. A697, 879 (2002); I. I. Balitsky and A. V. Belitsky, Nucl. Phys. B629, 290 (2002).

[33] O. Gituliar, M. Hentschinski, and K. Kutak, J. High Energy Phys. 01 (2016) 181; M. Hentschinski, A. Kusina, K. Kutak, and M. Serino, Eur. Phys. J. C 78, 174 (2018).

[34] R. Boussarie, A. V. Grabovsky, D. Y. Ivanov, L. Szymanowski, and S. Wallon, Phys. Rev. Lett. 119, 072002 (2017); R. Boussarie, A. V. Grabovsky, L. Szymanowski, and S. Wallon, J. High Energy Phys. 11 (2016) 149; G. Beuf, Phys. Rev. D 96, 074033 (2017); B. Ducloué, H. Hänninen, T. Lappi, and Y. Zhu, Phys. Rev. D 96, 094017 (2017); G. A. Chirilli, B. W. Xiao, and F. Yuan, Phys. Rev. D 86, 054005 (2012).

[35] M. Hentschinski, H. Weigert, and A. Schafer, Phys. Rev. D 73, 051501 (2006). 